\title{
Thyroid Gland Cavernous Hemangioma
}

National Cancer Institute

\section{Source}

National Cancer Institute. Thyroid Gland Cavernous Hemangioma. NCI Thesaurus. Code C156344.

A rare cavernous hemangioma that arises from the thyroid gland. 